\title{
HOMOLOGY MODELLING AND MOLECULAR DOCKING STUDY OF ORGANOPHOSPHATES AND PYRETHROIDS IN TERMS OF POTENTIAL TOXICITY
}

\author{
RAVI S. PANDEY ${ }^{* 1}$, RISHIKESH K. TIWARI ${ }^{1}$, SHIKHA SINGH ${ }^{1}$, SOMENATH GHOSH ${ }^{1}$, SUNIL K. RAI ${ }^{2}$, PRIYA SHREE ${ }^{3}$ \\ ${ }^{1}$ Biochemistry Laboratory, Department of Zoology, University of Allahabad, Allahabad 211002, India, Departments of ${ }^{2}$ Anatomy and \\ ${ }^{3}$ Medicinal Chemistry, Institute of Medical Sciences, Banaras Hindu University, Varanasi 221005, India \\ Email: rspandey2004@yahoo.com
}

Received: 26 May 2017 Revised and Accepted: 12 Jul 2017

\begin{abstract}
Objective: Though the adverse effects of pesticides used in agriculture may not immediately be visible in the human population however its long term exposure may cause detrimental effects by biomagnifications and bioaccumulation. Nowadays bioinformatics serves as an in silico tool not only for homology alignment but also for prediction of quaternary structures of biochemicals. The present study was aimed to compare the potential toxicities of triazophos and chlorpyrifos (organophosphates; OPs) and cypermethrin and deltamethrin (pyrethroids) and their interactions with cytochrome $\mathrm{P}_{450}$ functioning.
\end{abstract}

Methods: The authors performed the BLAST for homology alignment for cytochrome $\mathrm{P}_{450}$ of human and Zebra fish and further proceeded for docking analysis of all the pesticides with cytochrome $\mathrm{P}_{450}$.

Results: It was noted that $99 \%$ of query cover with $32 \%$ of homology in the sequences of cytochrome $\mathrm{P}_{450}$ between human and Zebra fish. Upon docking, the pesticide deltamethrin showed the highest interaction with cytochrome $\mathrm{P}_{450}$ with highest binding energy and least dissociation constant for Deltamethrin which was found to be 8.233 [kcal/mol] and 922849.687 [pM].

Conclusion: Our preliminary results thus encompass/indicate that the deltamethrin is not only having detrimental effect on enzyme kinetics in general but also such similar effects be apprehended for human also.

Keywords: Cytochrome $\mathrm{P}_{450}$, Homology modelling, Molecular docking, Organophosphate, Pyrethroids

(C) 2017 The Authors. Published by Innovare Academic Sciences Pvt Ltd. This is an open access article under the CC BY license (http://creativecommons.org/licenses/by/4.0/) DOI: http://dx.doi.org/10.22159/ijpps.2017v9i9.20285

\section{INTRODUCTION}

Synthetic pesticides of any origin can exert varied effects on different target as well as non-target organisms which further may also affect humans by biomagnification [1]. The organophosphate group of pesticides (like triazophos and chlorpyrifos) can directly affect the synapses present between neuronal and neuro-motor junctions by affecting acetylcholinesterase (AChE) activity [2]. The pyrethroids (like deltamethrin and cypermethrin) can also exert a similar effect by altering the voltage gated $\mathrm{Na}^{+}$ion channels present on neurolemma [3]. Thus, under both acute and chronic exposures, these pesticides are harmful and toxic to the aquatic biota particularly fishes [4] and further the human population as well due to the inability of detoxification of these pesticides [1]

The main detoxifying enzyme which is present in almost all cells ubiquitously as well as evolutionarily conserved one is cytochrome $\mathrm{P}_{450}$ (CYP) family proteins which use Haem as a cofactor for their functions [5]. Among a number of cytochrome $\mathrm{P}_{450}$ proteins, the mitochondrial cytochrome $\mathrm{P}_{450}$ is of the highest importance as it is primarily responsible for detoxifying drugs, drug metabolites, alcohol and others [6]. In human, there are about 57 genes reported for coding cytochrome $\mathrm{P}_{450}$ [7]. There are few reports, delineating the interactions of chlorpyrifos with cytochrome $\mathrm{P}_{450}$ in human, rat and mouse [8] but, till date reports are lacking in the field of homology alignment of cytochrome $\mathrm{P}_{450}$ in human and fishes and further the comparative interactions of cytochrome $\mathrm{P}_{450}$ with different pesticides.

The previous studies lacked the information on the structural homology of cytochrome $\mathrm{P}_{450}$ in human and a key representative of fish (Zebra fish; Danio rerio), therefore the present study was undertaken to note their similarities in functional activities. Further, the study was elaborated to note the interactions of pesticides (organophosphates and pyrethroids) with cytochrome $\mathrm{P}_{450}$ to get a speculative analogy of effects as exerted by these pesticides in fish are similar in human or not.

\section{MATERIALS AND METHODS}

Homology alignment of cytochrome $P_{450}$ between human and zebra fish

We compared the sequence of cytochrome $\mathrm{P}_{450}$ of Zebra fish and Homo sapiens using Basic Local Alignment Search Tool (BLAST).

\section{Molecular docking}

For in silico study, the protein structure of Cytochrome $\mathrm{P}_{450}$ with PDB ID: 4R21 were retrieved from RCSB protein databank [9]. Further, the geometry optimisation and active site prediction of this compound were done by using Discovery studio 3.0 [10]. The 2D structure of selected pesticides namely triazophos, deltamethrin, chlorpyrifos and cypermethrin were retrieved from Pubchem compound database [11] and converted into 3D format using Discovery studio 3.0. Then the best-docked compound was taken for interactive 2D-3D visualization using Discovery studio 3.0. Further molecular docking calculation was done by using YASARA software [12]. Using YASARA, receptors and ligands files were set and macro was run. The result log files were prepared for all the ligands. Binding energy and dissociation constant were used for sorting the docking result. The compound with more positive binding energy shows more interaction with the receptor.

\section{RESULTS}

Homology alignment of cytochrome $P_{450}$ between human and zebra fish

We found $99 \%$ query cover with $32 \%$ identity showing homology between human and zebra fish upon BLAST (fig. 1). 


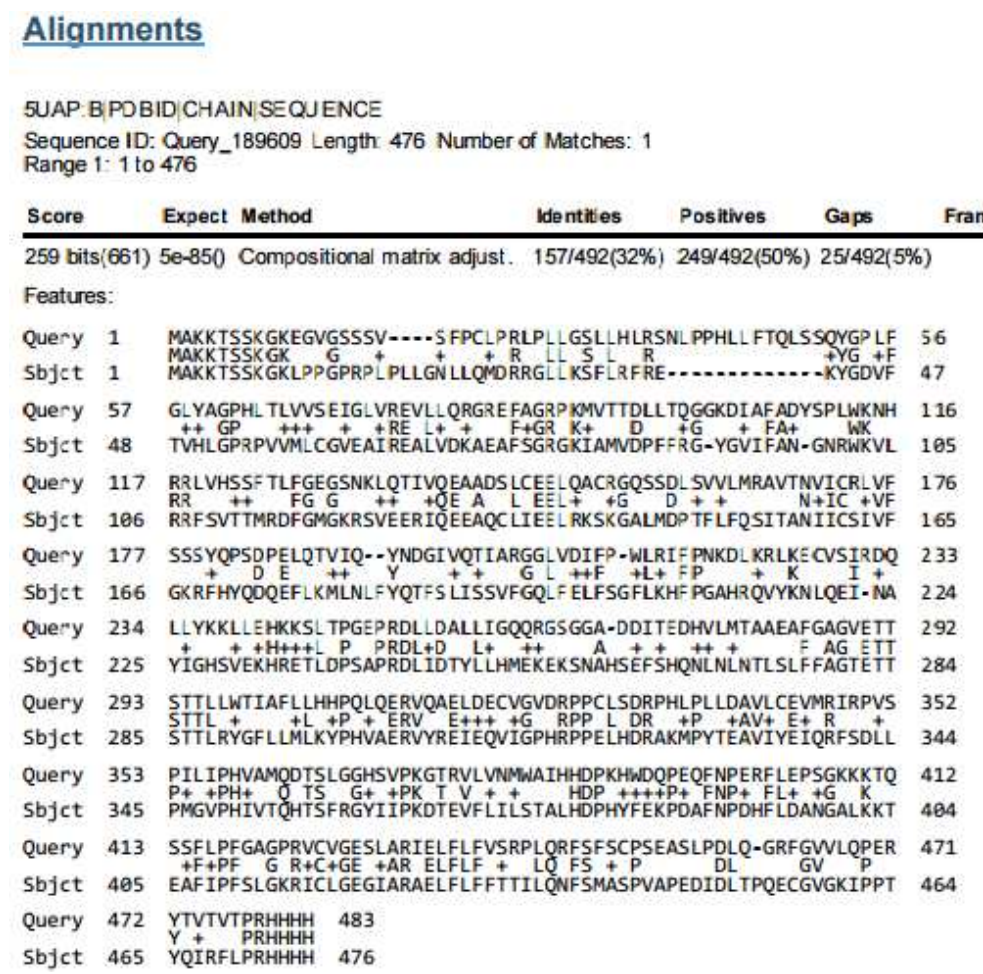

Fig. 1: Showing sequence homology alignment between human and zebra fish

\section{Molecular docking}

Molecular docking result showed that pesticide, deltamethrin exerted the best interaction with cytochrome $\mathrm{P}_{450}$. All other pesticides namely triazophos, chlorpyrifos and cypermethrin also shows interaction but lesser than deltamethrin (table 1). The binding energy and dissociation constant for deltamethrin were found to be $8.233[\mathrm{kcal} / \mathrm{mol}]$ and 922849.687 [pM] respectively. Further, the best-docked compound deltamethrin was taken for interactive 2D-3D visualization using Discovery studio 3.0. The active site amino acid residues, $\operatorname{ArgA}^{103} \operatorname{Ile}^{119} \operatorname{AlaA}^{120} \operatorname{TrpA}^{128}$ ArgA $^{132}$ AlaA $^{302}$ ThrA $^{306}$ ThrA $^{310}$ MetA $^{361}$ ValA $^{366}$ SerA $^{367}$ LeuA $^{370}$ IleA $^{371}$ HisA $^{373}$ ProA ${ }^{432} \quad$ PheA $^{433} \quad$ GlyA $^{434} \quad$ ArgA $^{438}$ ValA ${ }^{439}$ CysA $^{440}$ ValA ${ }^{441}$ GlyA $^{442}$ and $\mathrm{AlaA}^{446}$ of cytochrome $\mathrm{P}_{450}$ were involved in interaction with deltamethrin.

The pink colour residues show electrostatic interaction while green colour residues show Vander Waals interactions. The residue $\operatorname{ArgA}^{103}$ shows direct interaction as well as Pi-Pi interaction with deltamethrin which shows its inhibition activity (fig. 2A and fig. 2B).

Table 1: Binding energy and dissociation constant for all selected pesticides with cytochrome $P_{450}$

\begin{tabular}{lll}
\hline Pesticides & Cytochrome $\mathbf{P}_{450}$ & \\
\cline { 2 - 3 } & Binding energy [kcal/mol] & Dissociation constant [pM] \\
\hline Deltamethrin & 8.233 & 922849.687 \\
Cypermethrin & 7.533 & 3007722.75 \\
Triazophos & 6.497 & 17283666 \\
Chlorpyrifos & 5.433 & 104125760 \\
\hline
\end{tabular}

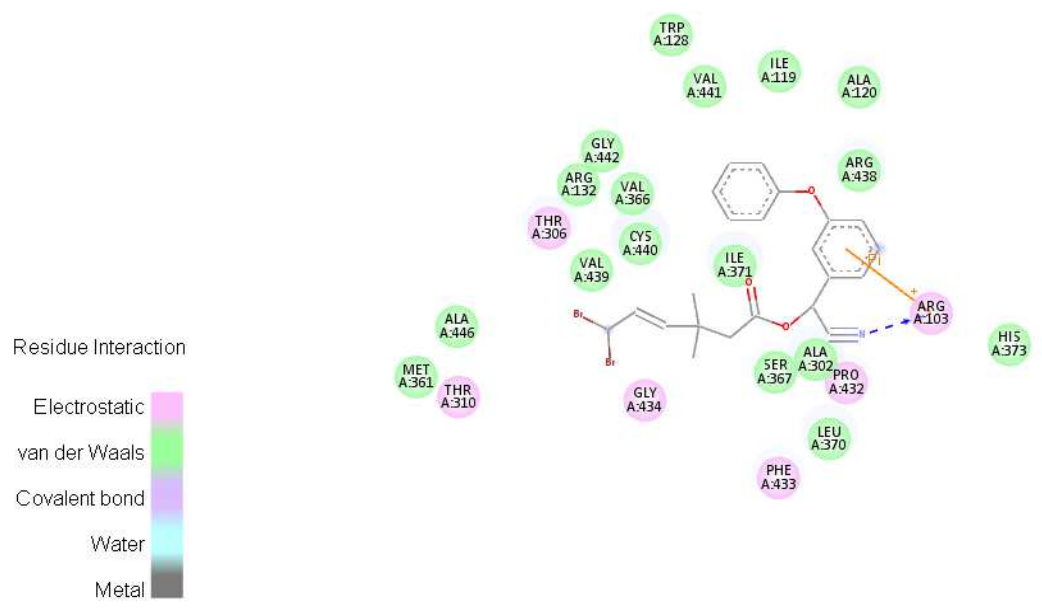

Fig. 2A: 2D interaction of deltamethrin with cytochrome $P_{450}$. The pink colour residues show electrostatic interaction while green colour residues show Vander Waals interactions. The residues $\mathrm{ArgA}^{103}$ shows direct interaction with deltamethrin 


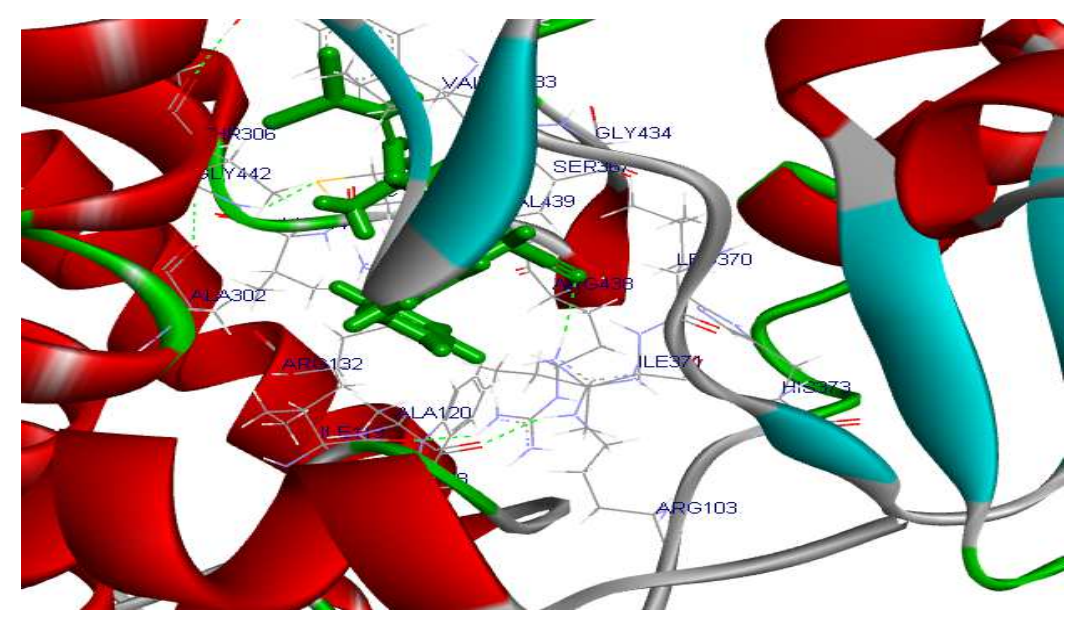

Fig. 2B: 3D interaction of deltamethrin with cytochrome $\mathbf{P}_{450}$

\section{DISCUSSION}

Fishes are the most economically important aquatic animals which are affected highly by pesticide contamination though being nontarget organisms [4]. The detrimental effect of pesticides can be ameliorated/detoxified by a key enzyme cytochrome $\mathrm{P}_{450}$. The interactions of cytochrome $\mathrm{P}_{450}$ with the pesticides like chlorpyrifos, triazophos, cypermethrin and deltamethrin individually and in combination has been analysed. It was noted that among four pesticides, the deltamethrin presented the highest binding with cytochrome $\mathrm{P}_{450}$ with highest binding energy and least dissociation constant. Thus, it may be speculated that, upon pesticide intoxication, deltamethrin can show the highest level of toxicity as it can directly bind with the active site of the enzyme with amino acid residues particularly with $\operatorname{ArgA}[10]^{3}$ by direct as well as $\mathrm{Pi}-\mathrm{Pi}$ interactions. Further, the toxicity effects would be more detrimental as these pesticides can also degenerate the basic histo-architecture of a number of tissues like gills, kidney and liver. The degeneration of these tissues in fishes can, in turn, affect severely other haematological and cell mediated immune parameters in fishes [13].

In the next step of the present study, the structural homology of cytochrome $\mathrm{P}_{450}$ of fishes with have been human was executed. For this, the known sequences of cytochrome $\mathrm{P}_{450}$ of fish (Zebra fish) and human from literature data base [14] was retrieved on 15. 05.2017 and the BLAST tool was run. Out of $99 \%$ of sequences, $32 \%$ of sequence homology between human and zebra fish was found. However similar studies of molecular docking of antidiabetic activity of cinnamon compounds have been reported earlier [15] but probably, these results for the first time have suggested that the structural homology of cytochrome $\mathrm{P}_{450}$ of human and zebra fish might have the same structure at the amino acid level and based on this structural homology it may be speculated that this enzyme might be performing the same function both in fishes and human.

\section{CONCLUSION}

From the present in silico study, it may be concluded that the potential toxicity in the terms of reaction kinetics and binding efficiency of two groups of pesticides namely organophosphates and pyrethroids are variable being higher in deltamethrin followed by cypermethrin, triazophos and chlorpyrifos respectively. Further, we can also speculate that the mode of action of these pesticides are almost similar even in human and fishes due to the structural homology of cytochrome $\mathrm{P}_{450}$ in both of the organisms.

\section{AUTHORS CONTRIBUTION}

Conceptualization of the study was suggested by RSP. The relevant literature survey and structural analysis of pesticides were done by RKT and SS (contributed equally). The analysis of results, writing of paper was done by SG. The docking analysis was done by SKR and PS.

\section{CONFLICT OF INTERESTS}

All the authors declare that they do not have any conflict of interest.

\section{REFERENCES}

1. Nelson JS. Fishes of the world. $4^{\text {th }}$ Ed. John Wiley and Sons; 2006.

2. Sanchez-Bayo FP. Insecticides mode of action in relation to their toxicity to non-target organisms. J Environ Anal Toxicol 2012;S4:1-11.

3. Narahashi T. Mode of action of pyrethroids. Bull World Health Org 1971;44:337-45.

4. Kumar A, Sharma B, Pandey RS. Preliminary evaluation of the acute toxicity of cypermethrin and lambda-cyhalothrin to Channa Punctatus. Bull Environ Contamin Toxicol 2007;79:613-6.

5. Sigel R, Sigel A, Sigel H. The ubiquitous roles of cytochrome $\mathrm{P}_{450}$ proteins: metal Ions in life sciences. Wiley and Sons; 2007.

6. Guengerich FP. Cytochrome $\mathrm{P}_{450}$ and chemical toxicology. Chem Res Toxicol 2008;21:70-3.

7. Human Genome Information Archive. "About the Human Genome Project". U. S. Department of Energy and Human Genome Project program; 2013.

8. Tang J, Cao Y, Rose RL, Brimfield AA, Dai D, Joyce A, et al. Metabolism of chlorpyrifos by human cytochrome P450 isoforms and human, mouse, and rat liver microsomes. Drug Metabol Dispos 2001;29:1201-4.

9. http://www.rcsb.org. [Last accessed on 15 May 2017]

10. Arora N, Singh VK, Shah K, Pandey-Rai S. Qualitative and quantitative analysis of 3D predicted arachidonate 15lipoxygenase-B (15-LOX-2) from Homo sapiens. Bioinformation 2012;8:555-61.

11. http://pubchem.ncbi.nlm.nih.gov. [Last accessed on 15 May 2017]

12. Krieger E, Vriend G. YASARA view-molecular graphics for all devices-from smart phones to workstations. Bioinfo 2014;30:2981-2.

13. Singh S, Tiwari RK, Ghosh S, Pandey RS. Impact of triazophos on Channa punctatus: hematological and histopathological studies. Int J Curr Res 2017;9:48246-52.

14. Hasemann CA, Kurumbail RG, Boddupalli SS, Peterson JA, Deisenhofer J. Structure and function of cytochromes P450:a comparative analysis of three crystal structures. Structure 1995;2:41-2.

15. Ganugapati J, Swarna S. Molecular docking studies of antidiabetic activity of cinnamon compounds. Asian J Pharm Clin Res 2014;7 Suppl 2:31-4.

\section{How to cite this article}

- $\quad$ Ravi S Pandey, Rishikesh K Tiwari, Shikha Singh, Somenath Ghosh, Sunil K Rai, Priya Shree. Homology modelling and molecular docking study of organophosphates and pyrethroids in terms of potential toxicity. Int J Pharm Pharm Sci 2017;9(9):9-11. 